\title{
Tumor immunotherapy: inching toward the finish line
}

\author{
Tyler J. Curiel ${ }^{1}$ and David T. Curiel ${ }^{2}$ \\ ${ }^{1}$ Hematology and Medical Oncology, Tulane Medical School, New Orleans, Louisiana, USA \\ ${ }^{2}$ Division of Human Gene Therapy, Departments of Medicine, Pathology, and Surgery, and the Gene Therapy Center, \\ The University of Alabama at Birmingham, Birmingham, Alabama, USA
}

Address correspondence to: David T. Curiel, Division of Human Gene Therapy, Departments of Medicine, Pathology, and Surgery, and the Gene Therapy Center, The University of Alabama at Birmingham, Birmingham, Alabama 35294-3300, USA.

Phone: (205) 934-8627; Fax: (205) 975-7476; E-mail: david.curiel@ccc.uab.edu.

J. Clin. Invest. 109:311-312 (2002). DOI:10.1172/JCI200215042.

Despite significant advances in therapy for early-stage cancer, the prognosis for most advanced-stage tumors remains little changed over the past 50 years (1). A vast array of novel strategies has been introduced over this period, many of them building on a recent explosion of insights into tumor immunology. Early attempts at cancer immunotherapy focused on IL-2 or other T cell-activating cytokines that were intended to expand the number or potency of $T$ cells specific for tumor-associated antigens (TAAs). Such activated T cells were expected to infiltrate the tumor and lead to the specific destruction of tumor cells. Although these attempts to boost antitumor immunity have yielded some limited examples of tumor regression, they have failed so far to control advanced-stage disease to a significant extent (2).

Effective $\mathrm{T}$ cell activation depends on the presentation of TAAs by antigen-presenting cells (APCs), such as macrophages, fibroblasts, B cells, or dendritic cells (DCs). Of these, only DCs can prime naive T cells, inducing their differentiation into antigen-specific effector cells. This feature, which was recognized early on, could potentially make DCs ideal agents for promoting TAA-specific immunity. Until recently, however, the rarity of these cells and the inability to cultivate them in large numbers hampered progress in this field. The development of techniques by which DCs can be differentiated ex vivo in large numbers revolutionized their study (3) and led to human trials that demonstrated the beneficial effects of TAAexpressing DCs, at least for some tumors $(2,3)$. The report of Heiser et al. in this issue of the JCI (4) now pro- vides additional encouraging clinical data that confirm the promise of DCbased immunotherapy.

\section{DC-based immunotherapy: variations on a theme}

The design of DC-based immunotherapies against cancer cells can vary in several respects. Key variables that will influence the safety and effectiveness of these therapies include the choice of TAAs, which may be either defined species or heterogenous mixes of different macromolecules; the technique of introducing the TAAs into DCs; and the preparation of the DCs themselves. Heiser et al. (4) settled on a relatively simple approach, introducing a single mRNA species directly into immature DCs, but it is useful to consider the range of alternatives that exist for each of these variables.

The TAAs chosen for use in immunotherapy may be either the products of mutated genes, such as mutant RAS or $p 53$, or normal self-antigens that are overexpressed or ectopically expressed in tumors. The use of normal cell surface antigens as TAAs can lead to undesired autoimmune responses. Thus, in the case of melanomas, TAAs might include proteins also expressed in skin and retina, and DC-mediated antimelanoma immunity may therefore depigment the skin, leading to vitiligo, or may impair visual acuity. Such complications have led some to question the wisdom of vaccinating against TAAs that are self-antigens, particularly if those self-antigens occur normally on critical organs such as the eye. The choice of the prostate-specific antigen (PSA), the antigen used by Heiser et al. (4), could circumvent this problem, since it is expressed primarily in a dispensable organ, the prostate.
In earlier trials, TAA presentation in DCs has generally been driven by application of synthetic peptides. While peptides are easy to make, other strategies may be advantageous, since peptides are expected to target only $\mathrm{CD}^{+}$or $\mathrm{CD}^{+} \mathrm{T}$ cells, rather than both together. The use of peptides also severely limits the epitopes expressed and the potential responding MHC haplotypes - a distinct limitation for clinical applications, given the diversity of MHC molecules in the human population. Further, a priori knowledge of protective epitopes is required, a problem that can be avoided by exposing the DCs to a more heterogenous set of proteins or nucleic acids (2, $3)$. One alternative is to expose DCs to tumor lysates or apoptotic bodies, thus allowing them to present a broad spectrum of TAAs. Ideally, this material should be derived from an autologous tumor, to ensure that it will include individualized TAAs. Another approach, transfection of DCs with recombinant DNA vectors encoding one or more potential TAAs, can clearly be effective, although the use of such vectors is associated with its own set of safety issues (5).

Heiser et al. (4) advance mRNA transfection as an answer to many TAA expression problems. mRNA may be derived from limiting amounts of autologous tumor. The heterogeneous population of RNAs from a given tumor would be expected to encode the complete set of relevant TAAs. In addition, application of mRNA to DCs apparently allows for the activation of both $\mathrm{CD}^{+}$and $\mathrm{CD}^{+} \mathrm{T}$ cells. In their current report, Heiser et al. have tested a simple version of this strategy, in which DCs are transfected with a single mRNA species, encoding PSA, but 
they note that a more general approach is also feasible. TAA expression might be increased by several variations on the direct application used here. For instance, liposomes could be used to facilitate mRNA delivery to the cells, or the mRNA could be stabilized by the addition of flanking sequences. The cells could also be modified to improve the efficiency of TAA processing and presentation or to induce the expression of accessory activation molecules. As the authors note, these modifications may be necessary in attempting to immunize against poorly immunogenic TAAs.

Another key variable in tumor immunotherapy is the source and differentiation status of the DC themselves. Heiser et al. (4) used immature monocyte-derived DCs, which have an excellent track record in human trials for safety and immunogenicity. Immature DCs derived from CD $34^{+}$cells have not been shown to be superior to these cells (6). Whether immature or mature DCs should be used, and what other features they should possess, is a matter of much debate, although mature DCs may be superior.

\section{Remaining concerns}

Heiser et al. confirm here that PSA mRNA induces immunogenic PSA expression on DCs, as evidenced by $\mathrm{T}$ cell proliferation (4). However, since tumors have proven to be adept at concealing their identity from the immune system, the crucial question remains whether this immune response leads to the lysis of autologous tumors. The authors note that they lacked sufficient autologous tumor to test this response directly, a problem that will clearly need to be addressed before the technique could be applied therapeutically. Moreover, especially given the absence of data on this clinical end- point, a detailed analysis of the cytokines induced by the treatment would be helpful in assessing the value of the treatment, as noncytolytic antitumor immunity is likely important.

Despite its apparent promise, adoptive DC immunotherapy still suffers from significant limitations. In addition to the clear possibility of inducing harmful autoimmune responses, there are equally daunting logistical problems related to the production of sufficient DCs. Heiser et al. used approximately $10^{8}$ DCs per patient over a period of several weeks (4). Ex vivo DC production is labor-intensive and expensive, requiring specialized and costly manufacturing facilities. If only $10 \%$ of the estimated 200,000 men who will develop prostate cancer in 2002 were eligible for adoptive DC immunotherapy, the incubator and freezer space required to accommodate these cells could quickly outstrip all available resources in the US. The requirements for recombinant cytokines are equally staggering. Clearly, adoptive DC immunotherapy may help establish certain proofs of concept, but means to make such technology tractable and cost-effective require much further thought.

Whether active immunization will ultimately be useful as an anticancer therapeutic strategy is also uncertain. Tumors are highly adept at downregulating TAAs or the cellular mechanisms required to present them. In a recent DC melanoma vaccine trial, patients who initially appeared to benefit from the therapy later relapsed, as their tumors had silenced the machinery to express the TAAs that could be recognized by peptide antigen-specific T cells (7). Anergy and tolerance deserve further consideration. For example, if a tumor tolerizes the immune system toward spe- cific TAAs, immunization will fail in the long run. In this regard, we recently demonstrated that in ovarian carcinoma, tumor-associated chemokines and DCs contributed to tumorspecific tolerance (8).

Despite all these caveats, Heiser et al. (4) provide two tantalizing suggestions that their approach may yet bear fruit. First, in some vaccinees, the rate of rise of PSA (PSA velocity) diminished. Second, in a smaller subset, circulating prostate cancer cells decreased immediately following therapy. These are encouraging data that require further investigation, as they suggest that vaccine-induced immunity was reducing tumor metastases. Nonetheless, it is clear that our increased understanding of tumor immunology is providing direct benefit in terms of therapeutic potential. Further advances in this field may be needed if the immunization procedures explored by Heiser et al. are ultimately to extend the survival of cancer patients.

1. Bailar, J.C., III, and Gornik, H.L. 1997. Cancer undefeated. N. Engl. J. Med. 336:1569-1574.

2. Rosenberg, S.A. 2001. Progress in human tumour immunology and immunotherapy. Nature. 411:380-384.

3. Banchereau, J., and Steinman, R.M. 1998. Dendritic cells and the control of immunity. Nature. 392:245-252.

4. Heiser, A., et al. 2002. Autologous dendritic cells transfected with prostate-specific antigen RNA stimulate CTL responses against metastatic prostate tumors. J. Clin. Invest. 109:409-417. DOI:10.1172/JCI200214364.

5. Balter, M. 2000. Gene therapy on trial. Science. 288:951-957.

6. Banchereau, J., et al. 2001. Immune and clinical responses in patients with metastatic melanoma to $\mathrm{CD} 34(+)$ progenitor-derived dendritic cell vaccine. Cancer Res. 61:6451-6458.

7. Schadendorf, D., and Nestle, F.O. 2001. Autologous dendritic cells for treatment of advanced cancer: an update. Recent Results Cancer Res. 158:236-248.

8. Zou, W., et al. 2001. Stromal derived factor-1 in human tumors recruits and alters the function of precursor plasmacytoid dendritic cells. Nat. Med. 7:1339-1346. 